\title{
RADAR OBSERVATIONS OF PLANETS WITH RT-70 PLANETARY RADAR IN EVPATORIA
}

\author{
Alexander I. Zakharov, Lyudmila N. Zakharova, Alexander S. Nabatov, Viktor P. \\ Sinilo, Mark V. Sorochinskii
}

Kotelnikov Institute of Radioengineering and Electronics of RAS, Fryazino Branch, http://fire.relarn.ru Fryazino 141190, Moscow Region, Russian Federation

aizakhar@ire.rssi.ru, zakharova@ire.rssi.ru,nabatov@ire.rssi.ru, sinilo@ire.rssi.ru, sorochinskii@ire.rssi.ru

Abstract. In 2012, after a 20-year break, radar observations of the planets in Evpatoria were resumed with a use of upgraded planetary radar with RT-70 antenna. One of the features of radar operations in 2012 was a new scheme of correction the Doppler distortion of sounding signal. The correction was implemented in digital form after wide band registration of the echo signal. This correction was performed in a form of monotonous adjustment of signal sampling frequency in accordance with nonlinear law of two-way delay variations estimated from the model of the planet motion with respect to radar. The sequence of further signal processing stages included chirp heterodyning, filtering into a narrow frequency band with reduction of the signal sampling frequency, calculation and incoherent accumulation of power spectra. The technique of the spectra analysis in the range measurements procedure utilized precalculated reference radiophysical spectra of the signal scattered by the planet. Measurements of the distance to Venus near the interior conjunction were made, and $3.8 \mathrm{~km}$ discrepancy with a forecast was found. Because of too large distance to Mars (more than 180 million $\mathrm{km}$ ), signal scattered by this planet was discovered only in one session with carrier signal. The results of radar observations confirm the operability of the RT-70 radar instrument in Evpatoria and ground-based signal processing software.

Keywords: radar astronomy, chirp signal, signal spectrum, planet orbit

UDC 621.396.967; 621.396.962

Bibliography - 4 references

Received 10.09.2018

RENSIT, 2018, 10(2):167-174

DOI: $10.17725 /$ rensit.2018.10.167

\section{Content}

1. INTRODUCTION (167)

2. The use of COMPLEX Signals IN THE RADAR OF PLANETS (168)

3. Mathematical model of the signal REFLECTED BY THE SURFACE OF THE PLANET (170)

4. The sequence of primary processing of THE PLANETARY RADAR (171)

5. The Results of RADAR MEAsurements IN 2012 (171)

6. Conclusion (173)

REFERENCES (173)

\section{INTRODUCTION}

Radar studies of the planets were started in the Institute of Nuclear Physics of the Academy of Sciences of the USSR in 1960 on the initiative of the director of the institute, Academician V.A. Kotel'nikov [1]. The use of radar methods in space research, which is the essence of radar astronomy, provides new opportunities for studying the celestial bodies of the solar system, new information on their position, motion, rotation parameters, dimensions, physical properties of the surface composing rocks, and much more. The increased practical interest in the development of radar methods in the interest of studying the planets in the early 1960s, among other things, was caused by the need to ensure the navigation of spacecraft during the planned interplanetary flights.

The most outstanding result of radar astronomy in the early 1960s was the acquisition and analysis of echoes from Venus, Mars, Mercury and Saturn. Pioneer work on radar 
observations of the planets of this period of observations was highly appreciated - a group of IRE staff members of the Academy of Sciences of the USSR, headed by Academician V.A. Kotelnikov (VM Dubrovin, VA Morozov, GM Petrov, ON Rzhiga, A. Shakhovskoy) received in 1964 the Lenin Prize.

Accumulation of the actual measuring material over the time interval from 1961 to 1980 made it possible to significantly clarify the parameters of the motion of the terrestrial planets and begin to create a new theory of planetary motion. The unified relativistic theory of the motion of the planets Earth, Venus, Mars and Mercury, created by the IRE Academy of Sciences of the USSR in collaboration with a number of other organizations on the basis of domestic and foreign radar and optical measuring material, made it possible to predict their relative position 50-100 times more accurately than the classical theory forecast movements of planets. The deviations of the measured distances from the planets from their calculated motion, calculated according to a unified theory, do not exceed the values: for Venus, $0.9 \mathrm{~km}$ in 1970-1980; for Mars - $2.5 \mathrm{~km}$ for 1967-1980; Mercury - $2.0 \mathrm{~km}$ in 1980. For a series of works on the creation of a unified relativistic theory of planetary motion, a group of Soviet scientists in 1982 was awarded the USSR State Prize. Among the awarded are the IRE staff of the USSR Academy of Sciences: Academician V.A. Kotel'nikov and head of the Laboratory of Radar Research of the Planets of the Institute of the Academy of Sciences of the USSR, G.M. Petrov.

\section{USE OF COMPLEX SIGNALS IN THE RADAR PLANET}

The method of measuring the planetary radar of the Center for Long-range Space Communication (CDNC) in Evpatoria in classical works on the radar of the planets of 1960-1980. was a well-established idea of using linear frequency modulation of the probe signal. The use of signals with complex modulation allows for a more detailed study of its surface by separating the signals reflected from the surface of the planet by delay and the Doppler frequency shift. The basis of the method is that selection by frequency allows you to isolate a signal reflected from a surface strip located at a certain distance from the axis of rotation of the planet, and selection by delay allows you to isolate the signal reflected from the annular band on the visible disk of the planet whose center coincides with the discontinuous point. The intersection of the bands of equal Doppler frequency shift and equal delay allows us to identify small areas on the planet's surface and achieve a high resolution on the surface, unattainable only due to the terrestrial antenna in the case of Venus from the Earth. As a rule, the sounding signal is a long premise of successive repeating probing pulses with intrapulse modulation (in our case - chirp), the duration of the signal on the radiation is equal to the time of the signal passing to the planet and back.

When probing the planet with a long radio pulse, its shape and carrier frequency are distorted due to the displacement of the reflecting surface during the arrival of the pulse. Specially created equipment made predistortions in the radiated signal so that at reception it was free from Doppler distortion of frequency and duration [2]. For this, the frequency of the emitted signal and the period of its sampling at the output of the LFM signal generator were continuously adjusted so that when receiving the signal it was at the nominal frequency and the duration of the chirp pulse was nominal and constant during the radar session. Chirping the receiving echo at the reception and further narrowband filtering made it possible to record the signal in a narrow band and successfully conduct further digital processing by then modest computing tools. Thus, the echo signal of Venus was digitized in the $4 \mathrm{kHz}$ band. The Mars signal was recorded 
in the $32 \mathrm{kHz}$ band. LFM heterodyning of the received signal at reception made it possible to simplify further processing, reducing it to calculation and accumulation of signal spectra, which did not require the construction of a multi-channel receiver for processing signals with different delay and frequency.

At the beginning of the work on the planetary radar in 2012, after almost a 20-year hiatus, the hardware of the planetary radar was profoundly modernized, as a result of which the technology of measurement and processing of measurements was changed, in particular, another method for correcting Doppler distortions of the signal was adopted. To clarify the features of the radiolocation of a new stage, let us consider the scheme for performing the measurements in Fig. 13]. If, at the moment of radiation, the radar is at point $\mathrm{A}$, the planet at the moment of arrival of the signal is at point $\mathrm{B}$, and the reception occurs at point $C$ (see), then the total retardation time $\tau$ will be equal to:

$\tau=0.5\left|r_{12}+r_{23}\right|$,

where $r_{12}$ and $r_{23}$ are the distances from the emitter to the planet and back, equal in turn:

$$
\begin{aligned}
& r_{12}=\left|\dot{r}_{2}\left(t_{2}\right)-\dot{r}_{1}\left(t_{1}\right)\right|, \\
& r_{23}=\left|\dot{r}_{3}\left(t_{3}\right)-\dot{r}_{2}\left(t_{2}\right)\right| .
\end{aligned}
$$

When using a monochromatic signal, the movement of the reflecting surface leads to a change in the frequency of the radio signal. Taking the relativistic effects as insignificant (the velocity of the planet is much less than

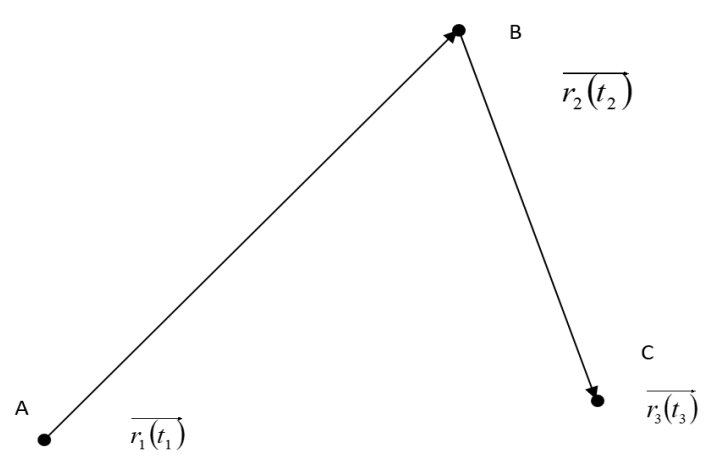

Fig. 1. Scheme of signal passage in the radar session of the planet. the speed of light), we find that the frequency of the signal arriving at the surface of the planet is

$$
f_{2}=f_{1}\left(1-\frac{V_{12}}{c}\right)
$$

where $f_{1}$ is the frequency of the radiated signal, $V_{12}$ is the relative velocity of the radar and the reflecting surface as the signal passes to the planet, $\mathrm{c}$ is the speed of light. The reflected signal comes to the radar with a frequency $f_{3}$ equal to

$$
f_{3}=\frac{f_{2}}{\left(1+\frac{V_{23}}{c}\right)},
$$

where $V_{23}$ is the relative velocity of the planet and the radar when the signal is received. The frequencies of the radiated and received signals are related by the following relation:

$f_{1}=f_{3} \frac{\left(1+\frac{V_{23}}{c}\right)}{\left(1-\frac{V_{12}}{c}\right)}=\eta f_{3}$.

The Doppler frequency shift at reception is

$$
f_{d}=f_{3}-f_{1}=f_{1}\left(1-\frac{1}{\eta}\right) \text {. }
$$

Consider the distortion of a narrow-band probing pulse, in which the carrier frequency $\mathrm{f}$ and the envelope $\mathrm{a}(\mathrm{t})$ can be distinguished. If the radiated pulse on the time scale of the radiator ttr can be described by expression

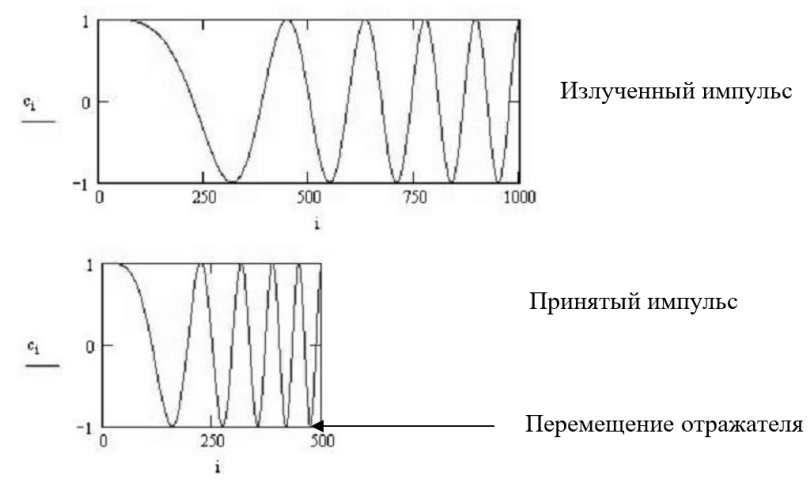

Fig. 2. Deformation of the probe pulse reflected by the moving object. 
$s_{\mathrm{tr}}\left(t_{\mathrm{tr}}\right)=a\left(t_{\mathrm{trt}}\right) \cos \left(2 \pi f_{1} t_{\mathrm{tr}}\right)$,

then on the receiving side the expression for the signal will have the form

$s_{\mathrm{r}}\left(t_{\mathrm{r}}\right)=a\left(t_{\mathrm{r}}\right) \cos \left(2 \pi f_{3} t_{\mathrm{r}}\right)$.

An example of distortion of a pulse with a chirp filling by a reflector approaching the radar is shown in Fig. 2. Here, along the abscissa, are the numbers of the signal samples.

The coefficient $\eta$ from (6) is actually the scale factor of deformation of the time structure of the probing signal:

$f_{1} t_{\mathrm{tr}}=\eta f_{3} t_{\mathrm{tr}}=f_{3} t_{\mathrm{r}}$

Therefore, by introducing a distortion in the time scale tr, we can compensate for the Doppler distortion of the pulse shape/ duration. Introducing a new time scale at the reception tint $=\operatorname{tr} \eta$, we obtain

$s_{\mathrm{r}}\left(t_{\mathrm{int}}\right)=a\left(\mathrm{t}_{\mathrm{r}} \eta\right) \cos \left(2 \pi f_{1} t_{\mathrm{r}} \eta\right)$.

A new algorithm for eliminating the Doppler effect was to emit a signal with fixed parameters and deform the time scale in the received signal. The deformation of the time scale was that the elementary step in time for sampling successive samples of the deformed signal was taken equal to dtrn, not to the interval between samples dtr. The number of the $k$-th count of the deformed signal is the integer part of $k \eta d t r$. Deformation of the timeline can be performed by skipping or duplicating some samples of the received signal. Note that $\eta$ is not a constant. It changes during the session due to changes in the relative velocities of the planet and the radar (see (6)).

Since skipping or duplicating samples leads to phase distortions, this effect should be minimized. For this, the frequency of the signal quantization in the receiving path must be significantly higher than the frequency band. Estimates show that, with an eightfold increase, phase errors with this time scale deformation method result in a signal/noise ratio (SNR) reduction of less than $1 \mathrm{~dB}$.
The next important point in eliminating the Doppler effect at the reception was the fact that before recording the signal in the receiving path is transferred from the carrier to some intermediate frequency in the lowfrequency region, and therefore, instead of (11), we write

$s_{\mathrm{r}}\left(t_{\mathrm{r}}\right)=a\left(t_{\mathrm{r}}\right) \cos \left(2 \pi\left(f_{3}-f_{\mathrm{g}}\right) t_{\mathrm{r}}\right)=a\left(t_{\mathrm{r}}\right) \cos \left(2 \pi f_{\mathrm{i}_{\mathrm{r}}}\right)$,

where $f g$ is the frequency of the heterodyne oscillation, and $f i$ is the intermediate frequency, in the simplest case containing the Doppler correction to the carrier. The correct sequence of actions includes, as a first stage, the transfer of a signal from frequency fi to zero frequency (elimination of Doppler by a carrier), and the second is the elimination of Doppler by the envelope a(tr).

\section{MATHEMATICAL MODEL OF SIGNAL, REFLECTED BY THE SURFACE OF THE PLANET}

The planet is an extended goal with a strongly intersected relief, and therefore the reflected signal has a rather complex shape, which makes it difficult to measure the distance to the planet. As noted above, the rotation of the planet around its axis leads to a Doppler blur of the reflected signal spectrum. In Fig. 3 shows the position of the components of the reflected signal in terms of range and frequency. Here, Doppler sub-spectra of different range zones follow one after the other along the axis of the delay. When a

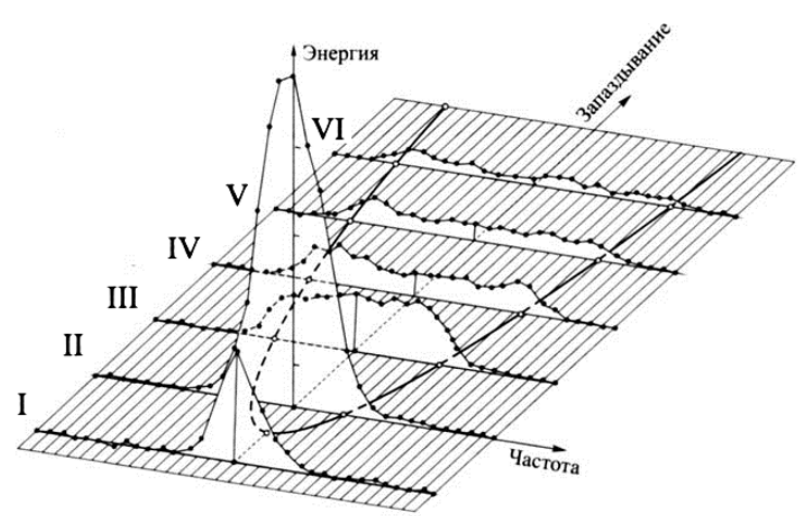

Fig. 3. Distribution of the reffected signal by delay and frequency. 


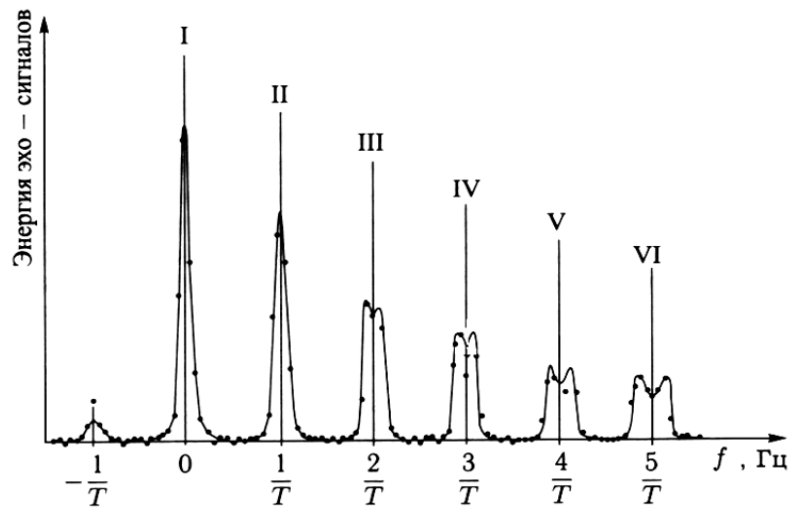

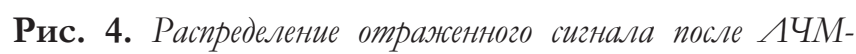
гетеродинирования на приеме.

processing scheme with LFM heterodyning and calculation of the signal spectrum is applied, the subspectra of different range zones are located on one frequency axis with a shift to the repetition rate of $1 / T$ pulses (see Fig. 4). Roman numerals in the figure indicate ranges of range 1-6 in Fig. 3.

Since the processing scheme with LFM heterodyning is the simplest in the computational plan, we take its basic one and give an expression for the spectrum of the model reflected by the surface of the planet when probing with periodically repeating pulses with a chirp filling.

The shape of the signal spectrum with linear frequency modulation (LFM signal) reflected by the surface of the planet and its position on the frequency axis are the basis for measuring the range, the velocity of the planet and the physical characteristics of its surface.

To obtain a mathematical model of the energy spectrum, we use the formula for the integral of elementary echoes over the surface of the planet, proposed by A.F. Khasyanov [4]:

$$
T(m)=B \iint_{S} \sigma_{0}(r, C) A_{m}(x, y) d s .
$$

Here $\mathrm{m}$ is the spectral reference number, $\mathrm{B}$ is a proportionality factor that takes into account the area of the radar antenna, the power and wavelength of the emitted signal, and the distance to the planet. Integration proceeds over the area of the resolution element s on the surface of the planet. Specific Effective Scattering Area where $r$ is the radius vector from the observation point to the point of the planet's surface, is described by the formula:

$$
\sigma_{0}(\theta(\vec{r}))=\frac{\rho_{0} C}{2}\left(\cos ^{4} \theta+C \sin ^{2} \theta\right)^{-1.5},
$$

where $\varrho_{0}$ is the specular reflection coefficient, $\mathrm{C}$ is the roughness parameter in the Hagfors formula describing the backscattering diagram of the planet's surface, $\theta$ is the angle of incidence of the radio wave on the planet's surface, $A_{k}(x, y)$ is the hardware function of the probing periodically repeating chirp signal in rectangular coordinates $\{x, y\}$ on the surface of the planet. A cumbersome expression for the hardware function can be found in [4].

\section{SEQUENCE OF PRIMARY PROCESSING OF SIGNALS OF PLANET RADAR}

The primary processing covers the steps of converting the reflected radar signal from the raw signal sample array to measuring the corrections to the delay and the speed of the probed planet, as well as the radiophysical parameters of the reflecting surface.

For the variant of shooting with pulse emission with fixed parameters, the first stage of processing after recording the echo was the elimination of signal distortion due to the Doppler effect (Doppler on the carrier and envelope). The next stage is chirp-heterodyning and filtering into a narrow frequency band. Next, the calculation and incoherent accumulation of power spectra, which is the input material for model processing, occurred. Extremely small values of the signal-to-noise ratio are characteristic for experiments on planetary radar, and therefore an obligatory stage of processing is incoherent accumulation of a mixture of signal power and noise and the subsequent subtraction of the noise pedestal. In our processing scheme, the form of the additive noise spectrum was approximated by 
a polynomial of the 12th degree, the smooth approximation was subtracted from the signalto-noise mixture. The models of the reflected signal spectra, calculated taking into account the survey parameters for the set of values of the Hagfors roughness parameter and the delay error, were used in the correlation procedure to determine the corrections to the ephemerides provided by the IPM RAS.

\section{RESULTS OF 2012 RADAR MEASUREMENTS}

The main series of work on the calibration of the equipment of the modernized planetary radar based on the RT-70 antenna and the development of radar measurement techniques was conducted during the period from 11 to 18 June 2012, in the region of the lower connection of Venus with the Earth. A month earlier, on April 27, a one-day series of radar observations of Venus and Mars was carried out as part of the testing of the software. On each of the days of observations, work was carried out on Venus and Mars.

A typical measurement session consisted of a radiation cycle, a reception cycle, and a pause needed to prepare for the next session. The sessions differed from each other by the duration of the radiation cycle, the object of measurement (Venus, Mars) and the type of the emitted signal (carrier, LFM signal with different parameters - deviation and repetition period). A total of about 50 sessions were conducted. The signal-to-noise ratio estimated from the radar-bearing sessions of Venus with the carrier was $\sim 30 \mathrm{~dB}$, which is close to the theoretical estimates for this planet at a distance of 44 million $\mathrm{km}$ with a Hagfors C roughness parameter of 400 , reflectivity of the surface $\varrho=0.05$ at a transmitter power of PRL $100 \mathrm{KW}$ and noise temperature of the receiving system is $30^{\circ}$.

Measurements of the range to Venus were conducted in sessions with a chirp signal with a deviation of 100, 200,400, $500 \mathrm{kHz}$ and a repetition period of 20 and $30 \mathrm{~ms}$. Since the unknown hardware delay of the transmitreceive path can be a source of errors in measuring the parameters of the motion of the planet, at the beginning of each session, a sounding signal was recorded in the "ring" mode. The hardware delay was calculated from the mutual position of the time of the onset of the formation of the chirp signal for radiation and the position of the chirp pulse during reception.

The results of the primary processing, the spectra of the reflected signal of Venus (Fig. 5) after correction of the Doppler effect and the chirp filtration are given below for various parameters of the probe signal. With the received processing parameters, the signal spectra in the $12 \mathrm{kHz}$ band with a resolution of $\sim 0.4 \mathrm{~Hz}$ were formed. Due to the high signal-to-noise ratio, a signal of several hundred zones can be observed in range. For greater clarity, we give only a portion of the spectrum near the nominal frequency, containing the first 10-15 zones in range. One can see a noticeable broadening of the far-band signal spectrum. The graph below shows the signal power in dimensionless units along the vertical axis, and the horizontal frequency in Hertz. An example of the combination of the real and model spectra for the session №7 from 11.06.12 is shown in Fig. 6. On the horizontal axis here are the numbers of the spectrum filters.

The solid line in Fig. 6 - model spectrum, discontinuous - the spectrum of the real signal. The difference in spectra is due to inaccuracies in the scattering parameters in the model, as well as

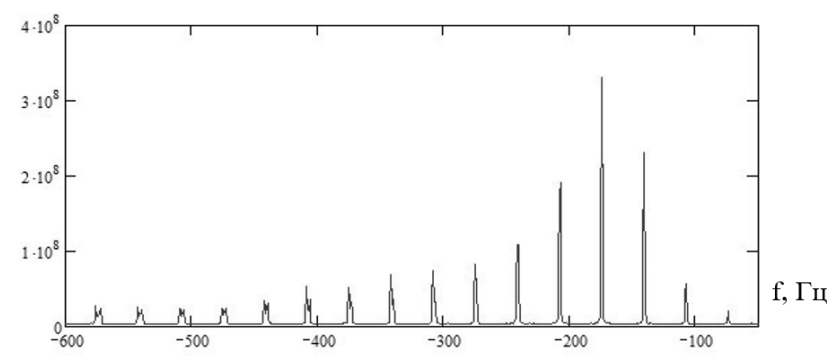

Fig. 5. Spectrum of the signal reflected by Venus LFM signal 11.06.12 in the session number 3 near the face value. The frequency deviation is $195 \mathrm{kHz}$, the period is $30 \mathrm{~ms}$. 


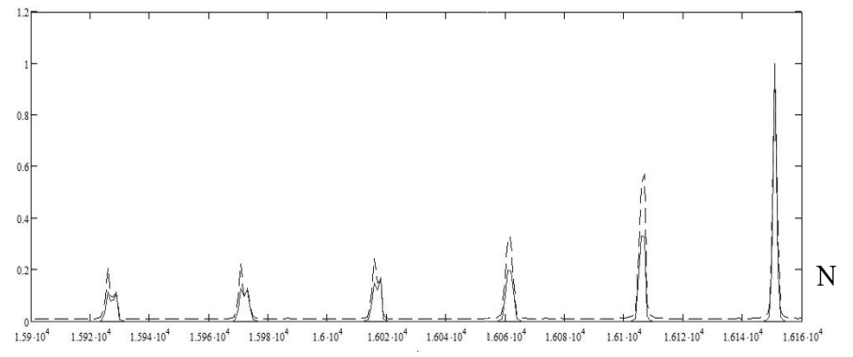

Fig. 6. Combination of real and model signal spectra for the session № 7 from 11.06.12.

by the features of the surface relief of the planet. In particular, the asymmetry of the band spectra in the left part of the graph is due to the presence of more strongly reflecting objects on one of the edges of the annular range zone. Model spectra were used to estimate the correction to the distance to the planet. To improve the accuracy of the delay measurements, the signal spectrum was compared with a set of model spectra of the signal reflected by the planet, calculated in advance for several values of the Hagfors parameter. For most range measurements, it is obtained that the surface of the planet is $3.8 \mathrm{~km}$ further than the predicted value.

In Fig. 7 shows the height profile of the surface of Venus relative to the mean local radius of $6051 \mathrm{~km}$ according to the data of Magellan satellite in the equatorial region in the longitude range $336^{\circ}-354^{\circ}$. The asterisks mark the position of the substitute point on June 11 (left) and June 13 (right).

It can be seen that on June 13, 2012, the tapping point hit the low relief area $0.6 \mathrm{~km}$ lower than the June 11th 2012 measurement, which made the measured range longer and, instead of

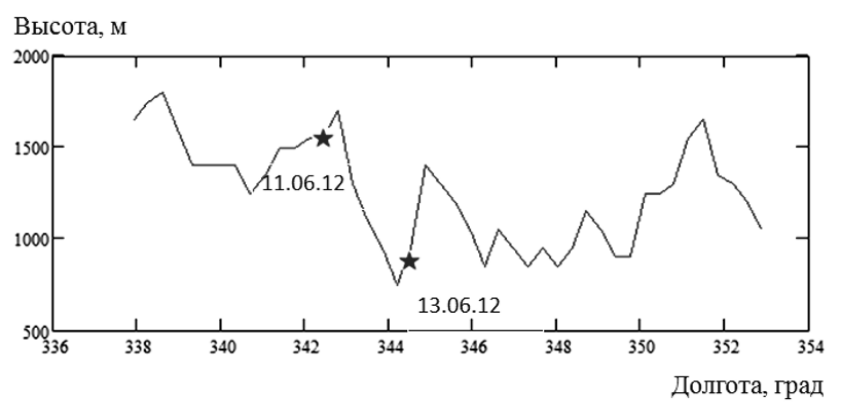

Fig. 7. Profile of the heights of the surface of Venus according to the data of the radar-profilograph of the Magellan spacecraft with the position of the substitute point in the sessions 11.06.2012 and 13.062012

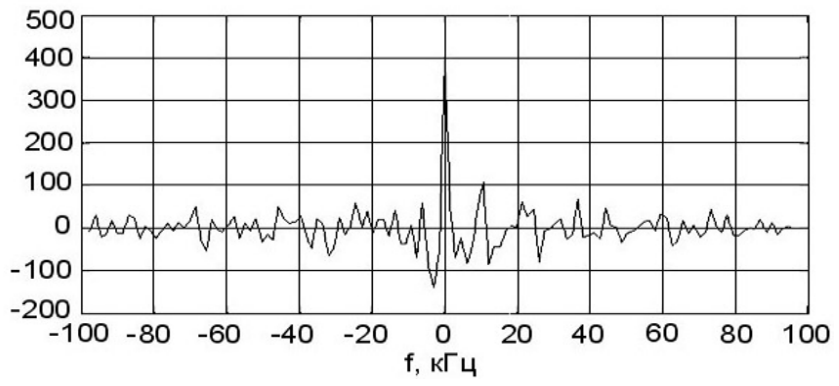

Fig. 8. Spectrum of the carrier signal after subtraction of noise for Mars in the session of 13.06.12.

$3.8 \mathrm{~km}$, measured $4.2 \mathrm{~km}$. Measured corrections to the range to Venus, predicted by the Institute of Applied Mathematics. M.V. Keldysh RAS (IPM), show that this planet in June was $3.8 \mathrm{~km}$ further than the forecast.

In the Mars radar sessions, a carrier and a chirp signal with a deviation of $32 \mathrm{kHz}$ and a repetition period of $1 \mathrm{kHz}$ were also used. Due to the long range to Mars in June 2012 (more than 180 million $\mathrm{km}$ ), the signal with a signalto-noise ratio of $\sim 10$, close to the theoretical one, was detected only in one session with the emission of the carrier signal. In Fig. 8 shows the spectrum of the echo signal from Mars with a frequency resolution of $800 \mathrm{~Hz}$, obtained after subtracting the noise pedestal in the form of a smooth approximation of a mixture of signal and noise by a polynomial of the 12th degree.

\section{CONCLUSION}

Work on the radar of Venus in 2012 was carried out using modernized planetary radar based on the RT-70 antenna in Evpatoria. In these works, a new scheme for correcting Doppler distortion of a signal was first used, which consisted in the fact that this type of distortion was eliminated after recording the echo signal in a wide band on the Earth. Measurements of the distance to Venus in the area of the lower connection with the Earth in June 2012 were obtained, which showed that the planet was at that time $3.8 \mathrm{~km}$ further than the predicted value. The results of the work confirmed the readiness of the equipment of the planetary radar and mathematical support for the processing of signals to regular work on planetary radar. 
174 ALEXANDER I. ZAKHAROV, LYUDMILA N. ZAKHAROVA,

\section{REFERENCES}

1. Kotelnikov VA. Collection of works. T.2. Space Radiophysics and Astronomy. M., Fizmatlit, 2009, 396 p.

2. Bakytko RV, Vasiliev MB, Vinitsky AS, Zaitsev AL, Grishmanovsky VA. Radio systems of interplanetary space vehicles. Moscow, Radio i svyaz Publ., 1993, 326 p.

3. Aleksandrov UN, Kuznetsov BI, Petrov GM, Rigga ON. Method of radar astrometric observations. Astronomich. Zhurnal, 1972, 49 (1): 175-185 (in Russ.).

4. Khasyanov AF. Radar studies of profiles of heights and reflective characteristics of the surfaces of Venus and Mars. Dis. Cand. fizmat. sciences. Moscow, 1981. 